\title{
Hyperoxic ventilation exacerbates lung reperfusion injury
}

Peter I. Ellman, MD, Jeffrey S. Alvis, MD, Carlos Tache-Leon, MD, Ramesh Singh, MD, T. Brett Reece, MD, John A. Kern, MD, Curtis G. Tribble, MD, and Irving L. Kron, MD

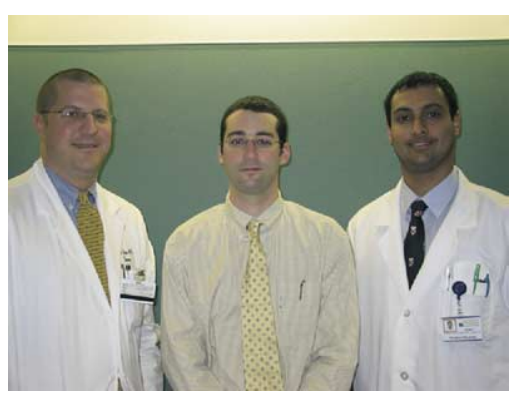

Reece, Ellman, Singh (left to right)

\section{Additional material} is available online.
From the Department of Cardiovascular Surgery, University of Virginia, Charlottesville, Va.

Supported by the National Institutes of Health under RO1 grant HL506093-03.

Received for publication April 15, 2005; revisions received June 7, 2005; accepted for publication June 16, 2005.

Address for reprints: Irving L. Kron, MD, 490 Lane Rd, Building MR4, Suite 3116, Charlottesville, VA 22908 (E-mail: ilk@ virginia.edu).

J Thorac Cardiovasc Surg 2005; 130:1440-5 $0022-5223 / \$ 30.00$

Copyright () 2005 by The American Association for Thoracic Surgery

doi:10.1016/j.jtcvs.2005.06.037
Objective: It is well known that hyperoxia can be potentially harmful to the ventilated patient, although little is known about the potential effects in the setting of lung reperfusion. We hypothesized that hyperoxic ventilation at the time of reperfusion could worsen the effects of lung reperfusion injury.

Methods: Using an ex vivo, blood perfused, isolated rabbit lung system, we evaluated the effects of hyperoxic (fraction of inspired oxygen $=100 \%, \mathrm{n}=10$ ) versus normoxic (room air, $\mathrm{n}=10$ ) ventilation after 18 hours of cold ischemia. Lungs were ventilated and perfused for 2 hours. A control group was immediately perfused and ventilated with a fraction of inspired oxygen of $100 \%$.

Results: Lung wet/dry ratios demonstrated lower tissue edema in the normoxic group compared with in the hyperoxic group $(6.72 \pm 0.89$ vs $7.62 \pm 1.14$ [mean \pm standard error of the mean], $P=.04$ ). Lung ventilation was also significantly better in the normoxic group versus the hyperoxic group $\left(\mathrm{PCO}_{2}=28.96 \pm 2.01\right.$ vs $36.68 \pm$ $3.20 \mathrm{~mm} \mathrm{Hg}, P=.04)$. Conversely, lung oxygenation after 2 hours of reperfusion (normoxic group ventilated for the last 15 minutes on 100\% fraction of inspired oxygen) was not significantly different between groups $\left(\mathrm{Po}_{2}=590.2 \pm 50.1\right.$ vs $499.6 \pm 67.5$ $\mathrm{mm} \mathrm{Hg}, P=.25$ ).

Conclusions: Ventilating lungs with $100 \%$ fraction of inspired oxygen at the time of reperfusion could increase the risk of lung reperfusion injury at the time of transplantation. Thus the patient should be ventilated with as low a fraction of inspired oxygen as possible to achieve adequate oxygen saturations during this critical reperfusion period.

I schemia-reperfusion (IR) injury in lung transplantation is manifested by nonspecific alveolar damage, lung edema, and hypoxia seen in the first 48 hours after lung transplantation. ${ }^{1}$ Although IR injury affects all transplanted grafts to some degree, $20 \%$ to $30 \%$ of the time it causes significant increases in mortality, time on a ventilator, intensive care unit and hospital stay, and costs. ${ }^{2}$ This injury has also been demonstrated to be a risk factor for bronchiolitis obliterans, the most common cause of long-term graft dysfunction. ${ }^{3}$

It is well known that hyperoxic ventilation can be harmful to the lungs. ${ }^{4-9}$ It is thought that hyperoxia causes its harmful effects in part because of the production of reactive oxygen species (ROS). ${ }^{9}$ ROS are also thought to be involved in IR injury. ${ }^{10-12}$ Thus it would logically follow that hyperoxia would potentially increase the substrate for the production of ROS in reperfusion injury and thus exacerbate the injury. It has been shown that hyperoxia exacerbates postischemic reperfusion injury in the heart, brain, and kidney. ${ }^{13-17}$ There are no studies, to our knowledge, that have examined the effects of hyperoxic ventilation in the setting of lung reperfusion injury. This issue is clinically relevant because it is common for lung transplant recipients to be ventilated with a fraction of inspired oxygen $\left(\mathrm{FIO}_{2}\right)$ of $100 \%$ at the time of reperfusion in the operating room. We hypothesized that 


$$
\begin{aligned}
& \text { Abbreviations and Acronyms } \\
& \begin{aligned}
\mathrm{ANOVA} & =\text { analysis of variance } \\
\mathrm{FIO}_{2} & =\text { fraction of inspired oxygen } \\
\mathrm{IR} & =\text { ischemia-reperfusion } \\
\mathrm{MPO} & =\text { myeloperoxidase } \\
\mathrm{PA} & =\text { pulmonary artery } \\
\mathrm{ROS} & =\text { reactive oxygen species }
\end{aligned}
\end{aligned}
$$

hyperoxic ventilation would exacerbate lung reperfusion injury.

\section{Materials and Methods \\ Experimental Protocol}

An ex vivo, blood perfused, isolated rabbit lung perfusion system was used for this experiment (Figure E1; Model TIS3862, Kent Scientific). Lungs were harvested en bloc with Viaspan (Belzer UW, Barr Laboratories Inc) preservation solution and placed on ice in a $4^{\circ} \mathrm{C}$ refrigerator for 18 hours. They were then reperfused for 2 hours. A control group was immediately reperfused on an $\mathrm{FIO}_{2}$ of $100 \%(\mathrm{n}=6)$. A second group was ventilated normoxically with room air $\left(\mathrm{FIO}_{2}=21 \%-24 \%\right)$ after 18 hours of cold ischemia $(\mathrm{n}=10)$. A third group was ventilated with a hyperoxic $\mathrm{FIO}_{2}$ of $100 \%$ after 18 hours of cold ischemia $(\mathrm{n}=10)$.

\section{Harvest Procedure}

New Zealand white rabbits of both sexes (3.0-3.5 kg) were randomly assigned to the 3 experimental groups. Each animal was anesthetized with intramuscular ketamine $(50 \mathrm{mg} / \mathrm{kg})$ and xylazine $(5 \mathrm{mg} / \mathrm{kg})$. Tracheal intubation was performed through a tracheostomy, and mechanical ventilation was instituted (Model RSP1002, Kent Scientific) with room air at a respiratory rate of 25 breaths/min. A median sternotomy and a thymectomy were then performed. The superior and inferior venae cavae were loosely encircled with ligatures, and the pericardium was opened. Both the pulmonary artery (PA) and the aorta were dissected free and similarly encircled. A purse-string suture was then placed in the free wall of the right ventricle, and intravenous heparin was administered (500 U/kg). After the PA was injected with $30 \mathrm{~g}$ of prostaglandin $\mathrm{E}_{1}$, the vena cavae were ligated to begin the period of ischemia. The PA was then cannulated through a right ventriculotomy in the center of the purse-string suture, and the right ventricular and PA ligatures were tied to secure the cannula. After the left ventricle was vented through a left ventriculotomy and the aorta was ligated, $50 \mathrm{~mL} / \mathrm{kg}$ cold $\left(4^{\circ} \mathrm{C}\right)$ Viaspan (Belzer UW, Barr Laboratories Inc) solution was infused into the PA from a height of $30 \mathrm{~cm}$. Topical cooling was achieved with cold saline solution slush. During the PA flush, the left atrium was cannulated through the left ventriculotomy with an outflow catheter. A purse-string suture was placed to secure this cannula. After completion of the PA flush, the inflow cannula was clamped. The lung-heart block was then excised, and the tracheostomy tube was clamped at end inspiration. The inflated lungs were immersed in cold saline solution and were stored at $4^{\circ} \mathrm{C}$. All animals received humane care in compliance with the "Guide for the care and use of laboratory animals" published by the National Institutes of Health (National Institutes of Health publication no. 85-23, revised 1985).

\section{Reperfusion Procedure}

After organ harvest and ischemic storage, the lung-heart block was suspended from a force transducer, and ventilation was initiated with either $100 \% \mathrm{FIO}_{2}$ or room air, depending on the experimental group (Harvard Ventilator). One breath of $30 \mathrm{~cm} \mathrm{H}_{2} \mathrm{O}$ of positive end-expiratory pressure was given once in the first 5 minutes of the stabilization period to ensure there was no atalectasis. Lungs were volume ventilated at 8 to $12 \mathrm{~mL} / \mathrm{kg}$ with $2.5 \mathrm{~cm} \mathrm{H} \mathrm{H}_{2} \mathrm{O}$ of positive end-expiratory pressure at a rate of 20 breaths/min. Volume was set such that peak inspiratory pressures would be $15 \mathrm{~cm} \mathrm{H}_{2} \mathrm{O}$ for all groups at 10 minutes. The volume would then not be changed for the remainder of the experiment. The lung-heart block was then connected through the PA catheter and the outflow catheters to a venous blood reperfusion circuit. New Zealand white rabbits served as fresh venous blood donors. Blood was circulated through a pediatric oxygenator set to deoxygenate the blood and add carbon dioxide. Thus the blood flowing through the PA was essentially physiologic for venous blood $\left(\mathrm{Po}_{2}=60 \mathrm{~mm} \mathrm{Hg} / \mathrm{PCO}_{2}=60 \mathrm{~mm} \mathrm{Hg}\right)$. This allowed arterial blood gases to be obtained from the left ventricle that reflected the physiologic performance of the lungs. Flow was gradually increased to $60 \mathrm{~mL} / \mathrm{min}$ over the first 5 minutes and then not changed for the remainder of the experiment.

\section{End Points: Physiologic Parameters}

Continuous recordings of pulmonary artery pressure and peak inspiratory airway pressure were performed by using a dynamic data acquisition program (DASYLab, DASYTEC) on a personal computer (Dell). This program automatically calculated and displayed PA pressures and peak inspiratory pressures every $5 \mathrm{sec}-$ onds throughout the experiment.

Pulmonary venous blood samples were collected for blood gas analysis (Corning $178 \mathrm{pH} /$ Blood Gas Analyzer, Corning, Inc) at $15,30,60$, and 120 minutes after the start of reperfusion. $\mathrm{Po}_{2} / \mathrm{FIO}_{2}$ ratios were calculated at these time points for comparison. At 1 hour and 45 minutes into the experiment, the $\mathrm{FIO}_{2}$ of the room air group was increased to $100 \%$ for the last 15 minutes of the experiment, allowing comparison of the oxygenation and ventilation by arterial blood gas at the 2-hour end point of the experiment.

\section{Tissue Analysis}

At the completion of the study, samples of the lower lobes of each lung were weighed and dried for calculation of wet/dry weight ratios. Lung samples were take from each lung and weighed immediately. The lung samples were then desiccated in an oven over 3 days. The ratio of lung dry weight to lung wet weight was then calculated as the wet/dry ratio. The average of the right and left lung were then used as the combined wet/dry ratio.

Samples of lung tissue were also taken from both lungs and frozen in liquid nitrogen and stored at $-80^{\circ} \mathrm{C}$ for later analysis of myeloperoxidase (MPO) activity. An MPO assay was performed on lung samples to quantify neutrophil sequestration in the same manner previously described by Ross and colleagues. ${ }^{18}$ Tissue was placed in $5 \mathrm{~mL}$ of $0.5 \%$ hexadecyltrimethylammonium bromide in $50 \mathrm{mmol} / \mathrm{L}$ potassium phosphate solution $(\mathrm{pH} \mathrm{7.4)}$ and disrupted by homogenizing at $4^{\circ} \mathrm{C}$. The solution was centrifuged at $15,000 \mathrm{~g}$ 


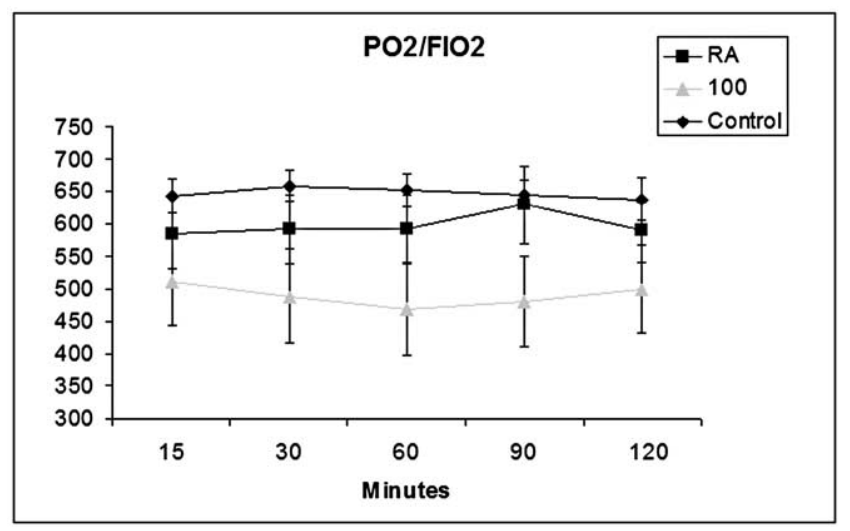

Figure 1. Time course of $\mathrm{Po}_{2} /$ fraction of inspired oxygen $\left(\mathrm{FiO}_{2}\right)$ in the control and ischemia-reperfusion groups (control group, $\mathbf{n}=$ 6; room air [RA] group, $\mathrm{n}=10 ; 100 \% \mathrm{FiO}_{2}$ group [100\%], $\mathrm{n}=10$ ). Values are presented as means \pm standard error of the mean. There were no significant differences between groups at any time point when the comparing $\mathrm{Po}_{2} / \mathrm{FIO}_{2}$ ratio. However, a trend toward a higher $\mathrm{Po}_{2} / \mathrm{FiO}_{2}$ ratio in the room air group when compared with the hyperoxic group can be seen at 90 minutes $(P=.08)$.

for 15 minutes at $4^{\circ} \mathrm{C}$, and the supernatant was discarded. The pellet was resuspended in $2 \mathrm{~mL}$ of $0.5 \%$ hexadecyltrimethylammonium bromide in $50 \mathrm{mmol} / \mathrm{L}$ potassium phosphate solution $(\mathrm{pH}$ 6.0) and homogenized. Tissue was disrupted further by means of sonication and then underwent 3 freeze-thaw cycles (liquid nitrogen bath $/ 37^{\circ} \mathrm{C}$ water bath). The solution was then centrifuged at $15,000 \mathrm{~g}$ for 15 minutes at $4^{\circ} \mathrm{C}$. Aliquots $(0.1 \mathrm{~mL})$ of supernatant were added to the assay buffer of $o$-dianisidine, $\mathrm{H}_{2} \mathrm{O}_{2}$, and 50 $\mathrm{mmol} / \mathrm{L}$ potassium phosphate ( $\mathrm{pH} 6.0)$. Absorbance at $460 \mathrm{~nm}$ was measured over 2 minutes by means of spectrophotometry (LKB Biochrom Model 4050). Lung tissue MPO activity was expressed as change in absorbance per gram per minute.

\section{Statistical Analysis}

The data are expressed as means \pm standard error of the mean. Statistical analysis was performed for the 3 groups by using analysis of variance (ANOVA), and post hoc analysis between groups was performed with the Fisher least significant difference test (SPSS 11.5, SPSS Inc).

\section{Results}

\section{Physiologic Parameters}

The PA pressures were significantly higher in the IR groups (room air and hyperoxic) compared with in the immediate control group at the initial portion of the experiment, as well as at 60 minutes (Figure E2; $P=<.001$, ANOVA at 0 minutes; $P=.025$, ANOVA at 60 minutes). There were no differences in the PA pressures between the room air group and the hyperoxic group. Peak inspiratory pressures were not different between the room air and hyperoxic groups, although they were significantly higher than in the control

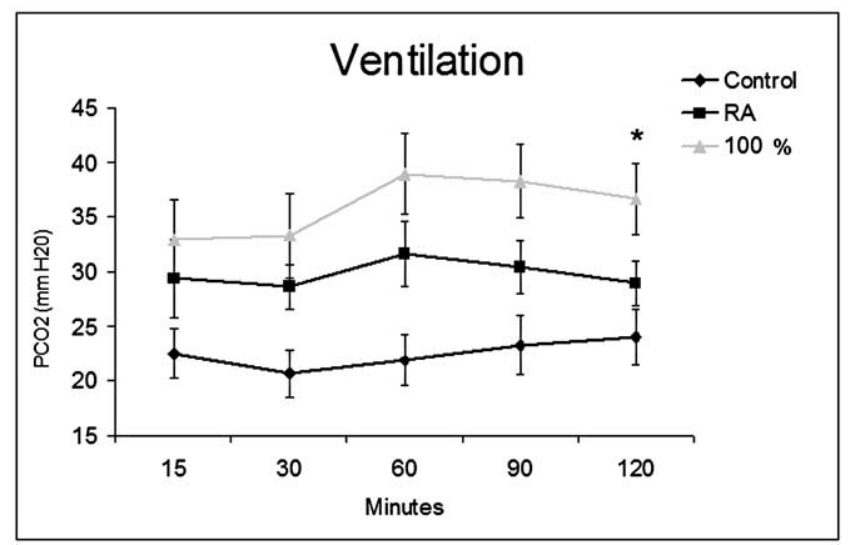

Figure 2. Time course of ventilation measured on the basis of $\mathrm{PCO}_{2}$ in the pulmonary venous blood gas in control and ischemiareperfusion groups (control group, $n=6$; room air [RA] group, $n$ $=10 ; 100 \% \mathrm{FiO}_{2}$ group [100\%], $\mathrm{n}=10$ ). Values are presented as means \pm standard error of the mean. Ventilation was significantly better in the room air group compared with in the hyperoxic group at 120 minutes $(P=.04)$.

group consistently throughout the first 60 minutes of the experiment (Figure E3).

There were no significant differences among all 3 groups with regard to oxygenation at 120 minutes (Figure E4). The $\mathrm{PO}_{2} / \mathrm{FIO}_{2}$ ratio showed no significant differences between groups at any time point in the experiment (Figure 1), although there was a strong trend toward poorer oxygenation in the hyperoxic group compared with the room air group at 90 minutes $(P=.08)$. The room air group demonstrated significantly better ventilation compared with the hyperoxic group at 120 minutes (Figure 2).

\section{Tissue Analysis}

The hyperoxic group demonstrated significantly higher levels of tissue edema when compared with the room air group, as well as the control group $(P=.002$, ANOVA; $P=.04$, hyperoxia vs room air; Figure 3 ).

There were no differences between groups with regard to tissue MPO levels, although there was a trend toward higher levels of tissue MPO in the hyperoxic group when compared with the room air group $(P=.11$, Figure 4$)$.

\section{Discussion}

It is well known that high $\mathrm{FIO}_{2}$ values during mechanical ventilation can be toxic to the lungs. However, the effects of hyperoxic ventilation in reperfusion injury, particularly in the lung, are not well defined. In the first 2 hours of reperfusion of cold ischemic lungs, we found that hyperoxic ventilation resulted in poorer ventilation and higher tissue edema when compared with that seen in normoxic venti- 


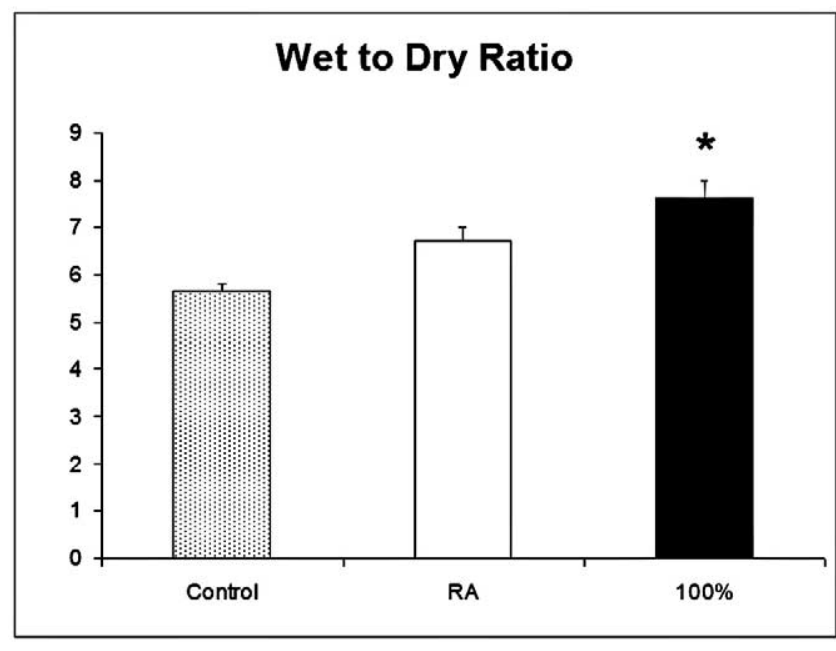

Figure 3. Lung edema measured on the basis of lung wet/dry ratios after 2 hours of reperfusion in the control and ischemiareperfusion groups (control group, $n=6$; room air [RA] group, $n$ $=10 ; 100 \% \mathrm{FiO}_{2}$ group [100\%], $\mathrm{n}=10$ ). Values are presented as means \pm standard error of the mean. Lung edema was significantly higher in the hyperoxic group compared with in the room air group. ${ }^{*} P=.002$, analysis of variance; $P=.04$, hyperoxia vs room air.

lated lungs. These findings are clinically relevant and suggest changes should be made in the operating room with regard to the $\mathrm{FIO}_{2}$ normally used during the time of reperfusion during lung transplantation. A review of the transplant database at the University of Virginia found that most (94\%) of the recipients receive $100 \% \mathrm{FIO}_{2}$ at the time of reperfusion in the operating room (data not published). However, the $\mathrm{Po}_{2} / \mathrm{FIO}_{2}$ ratios suggested about $70 \%$ of patients would have had adequate oxygenation on an $\mathrm{FIO}_{2}$ of $40 \%$. This suggests significant changes can be made safely with regard to lowering the $\mathrm{FIO}_{2}$ to more physiologic levels intraoperatively.

It has been previously shown that hyperoxia can exacerbate other forms of tissue injury. Smith and coworkers ${ }^{19}$ examined the effects of hyperoxia in the presence of acute lung injury. Interestingly, they found that the mortality for animals exposed to hyperoxia was significantly greater than for those breathing room air, regardless of the presence or absence of acute lung injury. Recently, Sinclair and associate $^{20}$ showed that hyperoxia exacerbates lung injury caused by lower $\mathrm{PaO}_{2} / \mathrm{FIO}_{2}$ ratios, increased lung injury scores, and increased polymorphonuclear leukocyte cell counts in the bronchoalveolar fluid. Hyperoxia might not cause worsening of myocardial injury because Shnier and colleagues ${ }^{21}$ found that hyperoxic reperfusion did not increase infarct size in an animal model of myocardial infarction.

A number of investigators have looked at the effects of hyperoxia during cardiopulmonary bypass and its effects on

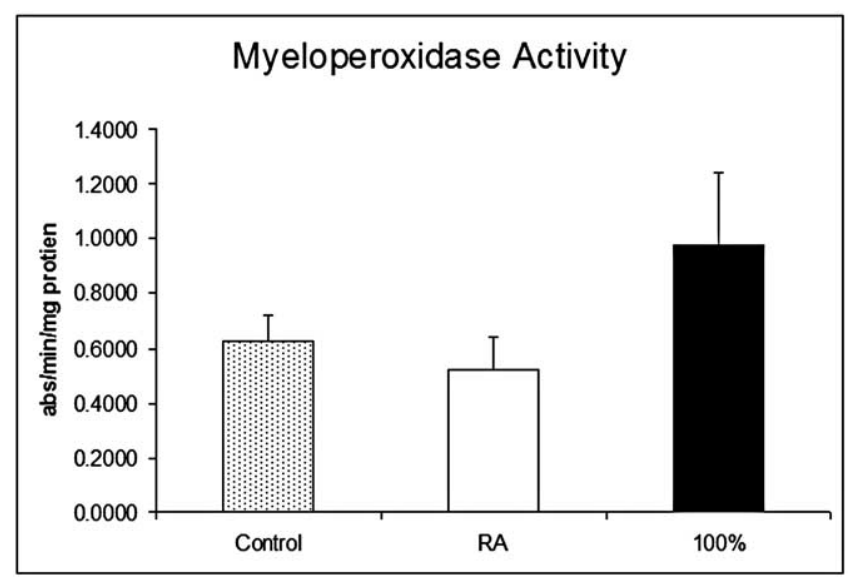

Figure 4. Myeloperoxidase activity in the control and ischemiareperfusion groups (control group, $n=5$; room air [RA] group, $n$ $=8 ; 100 \% \mathrm{FiO}_{2}$ group [100\%], $\mathrm{n}=9$ ). Values are presented as means \pm standard error of the mean. Myeloperoxidase activity was not significantly different between groups $(P=.11, \mathrm{RA}$ vs $100 \%)$.

the heart, as well as the brain. Ihnken and colleagues ${ }^{13}$ found that in immature piglet hearts normoxic cardiopulmonary bypass led to decreases in IR injury when compared with hyperoxic bypass. This was manifested by decreases in antioxidant reserve capacity and significantly poorer ventricular function when compared with that seen in a group that had been reperfused with normoxic cardiopulmonary bypass. In a clinical study Ihnken and colleagues ${ }^{14}$ demonstrated that hyperoxic bypass led to increased oxidative myocardial damage and poorer lung function evidenced by decreases in forced expiratory volume in 1 second and forced vital capacity. However, cardiac index was not different between groups in this particular study. Zwemer and coworkers ${ }^{17}$ demonstrated poorer neurological outcomes in animals who were hyperoxically reperfused on cardiopulmonary bypass after cardiac arrest when compared with normoxically reperfused animals. This effect was attenuated when an antioxidant was administered to animals who were hyperoxically reperfused. Liu and associates ${ }^{16}$ found similar results with regard to poorer neurological function in animals who were hyperoxically reperfused after cardiac arrest, as well as evidence for higher levels of oxidized brain lipids, suggesting higher levels of ROS in the hyperoxic group.

There is good evidence to suggest hyperoxia also exacerbates kidney IR injury. Sela and associates ${ }^{22}$ showed that postischemic reperfusion of the rat kidney with hyperoxic ventilation led to decreases in antioxidant enzyme activity, indirectly suggesting higher levels of ROS when compared with those present in the kidneys of normoxically ventilated animals. Zwemer and colleagues ${ }^{15}$ showed that hyperoxic reperfusion exacerbates postischemic renal dysfunction. 
This was manifested by increased plasma creatinine levels and urea nitrogen levels and higher histopathology injury scores in hyperoxically reperfused animals.

Splanchnic ischemia and reperfusion might be different from other organ systems with regard to the effects of hyperoxia during reperfusion after ischemia. Waisman and coworkers $^{23}$ found that hyperoxia in the setting of splanchnic ischemia and reperfusion led to an attenuation of inflammatory microvascular responses in both the mesenteric vasculature and the lung. It should be noted that in this particular study mean arterial blood pressure was significantly lower in the normoxically ventilated group, which suggests a possibility that mechanisms other than hyperoxia alone might have been the cause of these differences.

There are a few limitations to this study. Although we found statistically higher tissue edema and carbon dioxide retention in the hyperoxic group, there were no other significant differences between groups. It should be noted that we did not see significant IR injury in either the room air or hyperoxic groups. Previous studies in this laboratory with an isolated lung apparatus have demonstrated significant hypoxia, increased PA pressures, and tissue edema in nontreated IR groups. ${ }^{18,24} \mathrm{We}$ expected to see these pressures increase throughout the experiment when in fact we saw them diminish as the experiment progressed. The absence of significant reperfusion injury in either group might be due to 2 alterations that were made in the model for this particular study. The first was the addition of the deoxygenator. Previous studies in this laboratory used a trial of venous blood from outside the circuit to obtain arterial blood gases. We used a membrane oxygenator to deoxygenate and add carbon dioxide to the venous blood. We also used a gradual reperfusion-ventilation protocol to eliminate the possibility that lung injury was caused by endothelial shearing, nonphysiologic perfusion pressures, or barotrauma, rather than IR caused by the mechanisms that are proposed in the literature. ${ }^{1}$ The combination of these alterations might have attenuated the IR injury, and efforts to show differences between the room air and hyperoxic groups were blunted as well. Although significant differences were seen with regard to ventilation and lung edema, trends seen in $\mathrm{Po}_{2} / \mathrm{FIO}_{2}$ ratios and MPO levels might have reached statistical significance had the numbers been larger in the experimental groups. This study also did not perform a dose response, and thus the optimal $\mathrm{FIO}_{2}$ value cannot be determined on the basis of these data.

Peak inspiratory pressures and PA pressures were higher in the room air and hyperoxia groups compared with in the immediate control group. The fact that these differences were not present after 1 hour suggests they are due to the poor compliance that is caused by cold ischemia. Once the lungs had been warmed up to physiologic temperature, presumably they had similar compliance and vascular resis- tance, thus explaining the differences seen early in the experiment. Mechanistic studies were not performed, nor were any attempts to block the effects of hyperoxia performed with antioxidants to further support our hypothesis that the increased lung injury seen in the hyperoxic group was due to ROS. There was a strong trend toward higher $\mathrm{PO}_{2} / \mathrm{FIO}_{2}$ ratios at 90 minutes when comparing the room air with the hyperoxic group. Leaving the groups on the same oxygen tension throughout the experiment might have shown a significant difference at 120 minutes between groups, rather than trying to ventilate the room air group with an $\mathrm{FIO}_{2}$ of $100 \%$. Our study looked at the effects of hyperoxic ventilation on lung reperfusion injury in the first 2 hours of reperfusion. In the clinical setting lung reperfusion injury occurs over a 24- to 48-hour period, and for optimal clinical correlation, it would be advantageous to perform this study in a survival model of transplantation.

In conclusion, this is a unique study that examined the effects of hyperoxic ventilation on lung reperfusion injury in an isolated blood perfused model. This study is important because it has immediate clinical applicability in its demonstration that even in the first 2 hours of reperfusion hyperoxic ventilation exacerbates lung reperfusion injury manifested by higher levels of tissue edema and poorer ventilation. Our study suggests that high fractions of inspired oxygen should not be used in the operating room and perioperative period, and the lowest possible tension of inspired oxygen should be used during the time of reperfusion to reduce these deleterious effects.

\section{References}

1. de Perrot M, Liu M, Waddell TK, Keshavjee S. Ischemia-reperfusioninduced lung injury. Am J Respir Crit Care Med. 2003;167:490-511.

2. King RC, Binns OA, Rodriguez F, et al. Reperfusion injury significantly impacts clinical outcome after pulmonary transplantation. Ann Thorac Surg. 2000;69:1681-5.

3. Fiser SM, Tribble CG, Long SM, et al. Ischemia-reperfusion injury after lung transplantation increases risk of late bronchiolitis obliterans syndrome. Ann Thorac Surg. 2002;73:1041-8.

4. Freeman BA, Crapo JD. Hyperoxia increases oxygen radical production in rat lungs and lung mitochondria. J Biol Chem. 1981;256:1098692.

5. Bostek CC. Oxygen toxicity: an introduction. Aana J. 1989;57:231-7.

6. Zenri H, Rodriquez-Capote K, McCaig L, et al. Hyperoxia exposure impairs surfactant function and metabolism. Crit Care Med. 2004;32: 1155-60.

7. Griffith DE, Garcia JG, James HL, et al. Hyperoxic exposure in humans. Effects of 50 percent oxygen on alveolar macrophage leukotriene B4 synthesis. Chest. 1992;101:392-7.

8. Martin WJ 2nd, Kachel DL. Oxygen-mediated impairment of human pulmonary endothelial cell growth: evidence for a specific threshold of toxicity. J Lab Clin Med. 1989;113:413-21.

9. Jackson RM. Pulmonary oxygen toxicity. Chest. 1985;88:900-5.

10. Kennedy TP, Rao NV, Hopkins C, et al. Role of reactive oxygen species in reperfusion injury of the rabbit lung. J Clin Invest. 1989; 83:1326-35.

11. Fisher AB, Dodia C, Tan ZT, Ayene I, Eckenhoff RG. Oxygendependent lipid peroxidation during lung ischemia. $J$ Clin Invest. 1991;88:674-9. 
12. Adkins WK, Taylor AE. Role of xanthine oxidase and neutrophils in ischemia-reperfusion injury in rabbit lung. J Appl Physiol. 1990;69: 2012-8.

13. Ihnken K, Morita K, Buckberg GD, et al. Reduced oxygen tension during cardiopulmonary bypass limits myocardial damage in acute hypoxic immature piglet hearts. Eur J Cardiothorac Surg. 1996;10: 1127-35.

14. Ihnken K, Winkler A, Schlensak C, et al. Normoxic cardiopulmonary bypass reduces oxidative myocardial damage and nitric oxide during cardiac operations in the adult. $J$ Thorac Cardiovasc Surg. 1998;116: 327-34.

15. Zwemer CF, Shoemaker JL Jr, Hazard SW 3rd, et al. Hyperoxic reperfusion exacerbates postischemic renal dysfunction. Surgery. 2000;128:815-21.

16. Liu Y, Rosenthal RE, Haywood Y, et al. Normoxic ventilation after cardiac arrest reduces oxidation of brain lipids and improves neurological outcome. Stroke. 1998;29:1679-86.

17. Zwemer CF, Whitesall SE, D'Alecy LG. Cardiopulmonary-cerebral resuscitation with $100 \%$ oxygen exacerbates neurological dysfunction following nine minutes of normothermic cardiac arrest in dogs. Resuscitation. 1994;27:159-70.
18. Ross SD, Tribble CG, Gaughen JR Jr, et al. Reduced neutrophil infiltration protects against lung reperfusion injury after transplantation. Ann Thorac Surg. 1999;67:1428-34.

19. Smith RA, Venus B, Masood S, Carter MC. Effects of hyperoxia in the presence of acute lung injury. Crit Care Med. 1990;18:198-202.

20. Sinclair SE, Altemeier WA, Matute-Bello G, Chi EY. Augmented lung injury due to interaction between hyperoxia and mechanical ventilation. Crit Care Med. 2004;32:2496-501.

21. Shnier CB, Cason BA, Horton AF, Hickey RF. Hyperoxemic reperfusion does not increase myocardial infarct size. Am J Physiol Heart Circ Physiol. 1991;260:H1307-12.

22. Sela S, Shasha SM, Mashiach E, et al. Effect of oxygen tension on activity of antioxidant enzymes and on renal function of the postischemic reperfused rat kidney. Nephron. 1993;63:199-206.

23. Waisman D, Brod V, Wolff R, et al. Effects of hyperoxia on local and remote microcirculatory inflammatory response after splanchnic ischemia and reperfusion. Am J Physiol Heart Circ Physiol. 2003;285: H643-52.

24. Ross SD, Kron IL, Parrino PE, et al. Preservation of intercostal arteries during thoracoabdominal aortic aneurysm surgery: a retrospective study. J Thorac Cardiovasc Surg. 1999;118:17-25. 


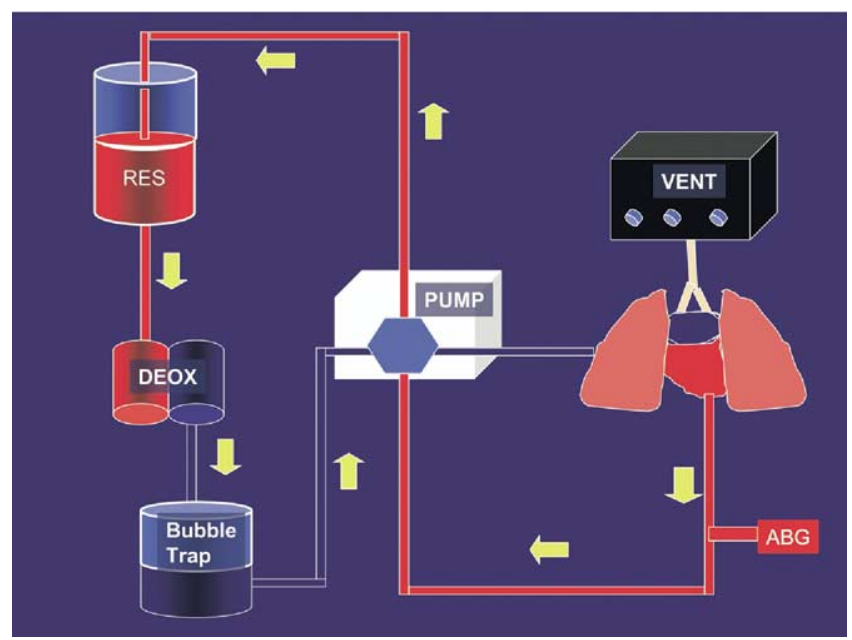

Figure E1. A diagram of the blood perfused isolated lung apparatus. RES, reservoir; DEOX, deoxygenator; $A B G$, arterial blood gas.

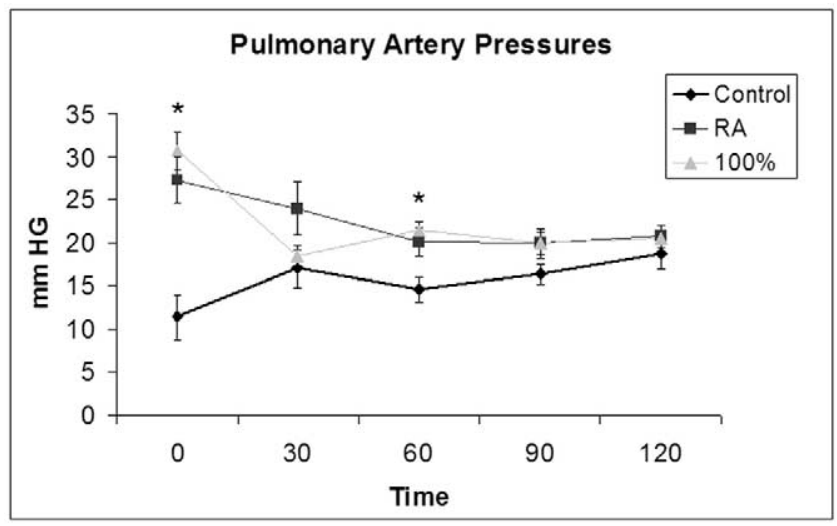

Figure E2. Time course of mean pulmonary artery pressures in the control and ischemia-reperfusion groups (control group, $\mathrm{n}=5$; room air $[R A]$ group, $\mathrm{n}=10 ; 100 \% \mathrm{~F}_{2} \mathrm{z}_{2}$ group [100\%], $\mathrm{n}=10$ ). Values are presented as means \pm standard error of the mean. Mean pulmonary artery pressures were not significantly different between the hyperoxic and room air groups. The hyperoxic and room air groups were significantly different compared with the control group at 0 and 60 minutes ${ }^{*} P \leq .001$, analysis of variance at 0 minutes; $P=.025$, analysis of variance at 60 minutes. 


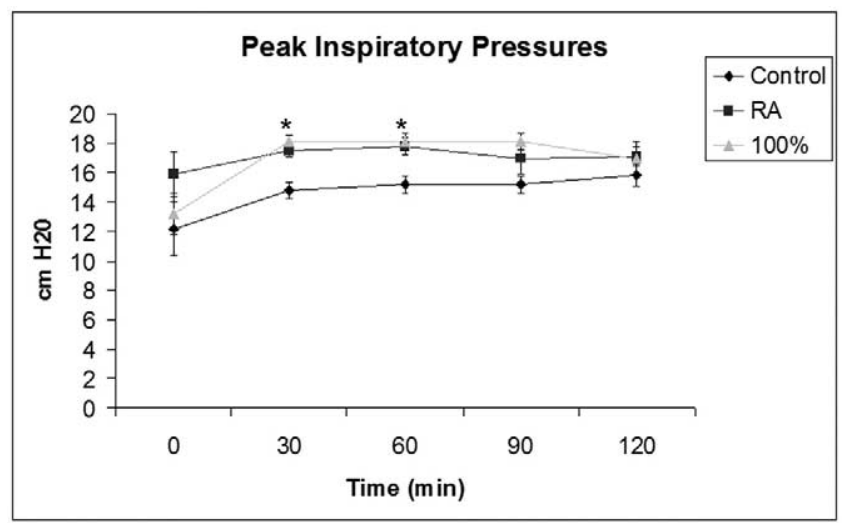

Figure E3. Time course of peak inspiratory pressures in the control and ischemia-reperfusion groups (control group, $n=5$; room air $[R A]$ group, $n=10 ; 100 \% \mathrm{FiO}_{2}$ group $[100 \%], n=10$ ). Values are presented as means \pm standard error of the mean. Peak inspiratory pressures were not significantly different between the hyperoxic and room air groups. The hyperoxic and room air groups had significantly higher peak inspiratory pressures compared with the control group at 0,30 , and 60 minutes.

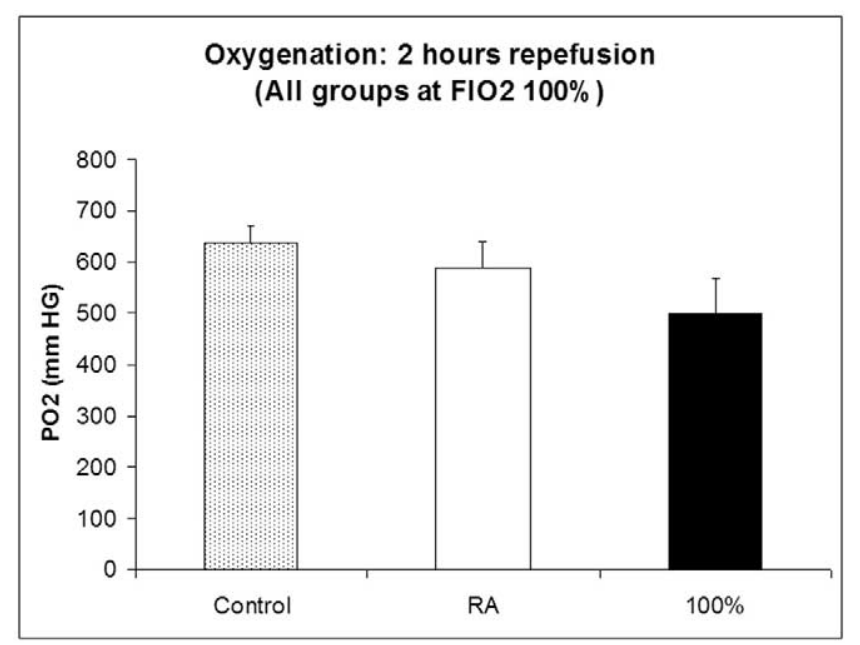

Figure E4. Oxygenation by arterial blood gas at 120 minutes (control group, $n=6$; room air [RA] group, $n=10 ; 100 \%$ $\mathrm{FiO}_{2}$ group $[100 \%], \mathrm{n}=10$ ). Values are presented as means \pm standard error of the mean. Oxygenation was not different between groups when comparing $\mathrm{PO}_{2}$ on a fraction of inspired oxygen $\left(\mathrm{FiO}_{2}\right)$ of $100 \%$ for all groups. The room air group received an $\mathrm{FiO}_{2}$ of $100 \%$ for the last 15 minutes of the experiment. 